\title{
APPARENCES ET RÉALITÉS DE LA RÉFORME DE L'ÉTAT BELGE
}

\section{Franklin Dehousse}

CRISP | « Courrier hebdomadaire du CRISP »

1986/33 $\mathrm{n}^{\circ} 1138$ | pages 1 à 38

ISSN 0008-9664

Article disponible en ligne à l'adresse :

https://www.cairn.info/revue-courrier-hebdomadaire-du-crisp-1986-33-page-1.htm

Distribution électronique Cairn.info pour CRISP.

(c) CRISP. Tous droits réservés pour tous pays.

La reproduction ou représentation de cet article, notamment par photocopie, n'est autorisée que dans les limites des conditions générales d'utilisation du site ou, le cas échéant, des conditions générales de la licence souscrite par votre établissement. Toute autre reproduction ou représentation, en tout ou partie, sous quelque forme et de quelque manière que ce soit, est interdite sauf accord préalable et écrit de l'éditeur, en dehors des cas prévus par la législation en vigueur en France. Il est précisé que son stockage dans une base de données est également interdit. 


\title{
Apparences et réalités \\ de la réforme de l'Btat belge
}

\author{
Franklin Dehousse
}

1. Le contrôle des relations internationales

2. La suprématie fiscale de l'Etat

3. La suprématie financière de l'Etat

4. La construction des règles de compétence

5. La superposition des règles de compétence 
Au cours des vingt dernières années, la Belgique a connu d'importantes mutations institutionnelles. En 1970, la troisième révision de la Constitution a consacré l'existence des Communautés et des Régions. Peu après, le vote de la loi spéciale du 21 juillet 1971 a permis l'entrée en fonction des Conseils culturels. La mise sur pied des institutions régionales a été plus ardue. Elle a été entamée par la loi de régionalisation préparatoire du ler août 1974. Elle a été poursuivie par les lois du 19 juillet 1977 et du 20 juillet 1979. Finalement, la loi spéciale de réformes institutionnelles du 8 août 1980 et la loi ordinaire du 9 août 1980 ont modifié de manière importante à la fois la structure et le rôle des Communautés et des Régions (1).

La réforme de l'Etat belge a été présentée de diverses manières. Pour les uns, elle instaure un système sui generis, qui ne se rattache à aucune structure constitutionnelle connue. Plusieurs caractéristiques plaident en faveur de cette analyse : l'absence de primauté des normes nationales sur les normes communautaires ou régionales, la superposition sur un même territoire des Communautés et des Régions, le sort (ou l'absence de sort) destiné à l'entité de Bruxelles. Pour les autres, la réforme de l'Etat belge s'apparente à un système fédéral comportant des modalités particulières. D'autres caractéristiques plaident en faveur. de cette seconde analyse : l'autonomie de l'Etat, des Communautés et des Régions, ainsi que l'exclusivité de leurs compétences.

La nouvelle structure de l'Etat belge correspond-elle à un système fédéral? Depuis quelques années, les déclarations politiques invoquant "la coopération fédérale", "la bonne foi fédérale", "l'importance des juridictions dans le système fédéral belge" abondent. Toutefois, en dépit du vocabulaire

(1) Pour une blbllographle sommaire relative à la réforme de l'Etat, volr $F$. Dehousse, "Les conflits budgétaires dans la réforme de l'Etat", Courrler Hebdomadaire du CRISP, $n^{0} 1124-1125,5$ septembre 1986, notes 2, 3 et 4. 
politique actuel, la question posée appelle une réponse négative, à la fois pour des raisons historiques et juridiques. D'une part, l'histoire parlementaire des vingt dernières années atteste que les références à un hypothétique modèle fédéral ont été fort rares tout au long du processus de réforme institutionnelle. D'autre part, les premières années d'application de la réforme de l'Etat ont montré le caractère théorique de l'autonomie et de l'exclusivité de compétence des Communautés et des Régions.

Ces deux principes apparaissent largement liés l'un à l'autre.

- L'autonomie des Communautés et des Régions procède d'un ensemble d'éléments. Elles ne se trouvent pas soumises à un pouvoir de tutelle. Elles disposent d'institutions dans la désignation desquelles l'Etat n'intervient pas (2). La loi et le décret jouissent d'une force juridique équivalente. Les compétences et les institutions des Communautés et des Régions ont, de façon générale, été définies dans une loi à majorité spéciale, ce qui signifie qu'elles ne peuvent être modifiées sans l'approbation d'une majorité dans chaque groupe linguistique.

- La structure de la réforme de l'Etat se trouve néanmoins compliquée par le fait que certaines caractéristiques institutionnelles et certaines compétences des Communautés ont été définies par l'article 59bis de la Constitution. La loi spéciale du 8 août 1980 énonce seulement une partie des compétences communautaires. Cette technique a permis de doter les Communautés de prérogatives internationales plus importantes. Par contre, elle rend la réforme d'une partie du statut des Communautés plus difficile. La modification de l'article 59bis de la Constitution impose l'adoption d'une déclaration de révision et la dissolution des Chambres, alors que la modification de la loi spéciale du 8 aoút impose un vote à la majorité spéciale, mais sans déclaration de révision préalable.

L'exclusivité des compétences nationales et culturelles avait été établie en 1970, lors de la troisième révision de la Constitution. L'extension du principe aux compétences régionales a été soulignée dans le premier projet de réformes institutionnelles du 11 juillet 1978, au début du processus qui devait s'achever avec le vote de la loi spéciale du 8 aoot

(2) Les événements ont toutefols montré que le Rol et le gouvernement n'avalent pas été complètement écartés de la procédure de désignation : volr sur ce polnt F. Dehousse, "Le feullleton constltutlonnel de l'hiver : la désignation de l'exécutlf réglonal wallon", Journal des Trlbunaux, 1986, pp. 153-156. 
1980. A cette époque, le Conseil d'Etat avait d'ailleurs estimé que l'instauration d'un système de compétences concurrentes entre l'Etat et les Communautés réclamerait une révision constitutionnelle ( 3 ).

L'attribution aux décrets régionaux d'une force équivalente à celle de la loi, suite au vote de l'article 26 bis de la Constitution et de l'article 19 de la loi spéciale du 8 aout 1980, a étendu ce raisonnement aux compétences régionales. L'équipollence des normes nationales, comunautaires et régionales exclut toute idée de primauté entre elles et, partant, toute notion de concurrence (4).

Les premières années d'application de la réforme de l'Etat ont néanmoins montré le caractère théorique des principes d'autonomie et d'exclusivité des compétences. La description du nouveau paysage institutionnel belge s'est révélée, à I'examen, quelque peu incorrecte ou, plus exactement, incomplète. Cela-provient d'abord d'une fixation excessive, dans les milieux parlementaires et juridiques, sur les aspects normatifs de la réforme de l'Etat : la mythologie de la lai et l'équipollence des normes ont contribué a fausser les analyses. En réalité, la réforme de l'Etat belge a instauré, grâce à plusieurs mécanismes distincts, une répartition de compétences à géométrie variable. Les mécanismes en question donnent au pouvoir central la possibilité d'intervenir sans grandes difficultés dans les matières communautaires et régionales. Ils permettent, par ailleurs, l'établissement d'une forme de tutelle indirecte au profit de l'Etat.

(3) Doc. Parl., Sénat, sess. extr. 1979, n²61/1, annexe 11, pp. 31-32.

(4) "Les pouvoirs natlonaux, réglonaux ef communautaires ont des domaines de compétence exclusifs. Même lorsque différents pouvolrs territorlaux sont compétents pour la même matlère, Ils ne peuvent pas l'être de manlère concurrente, mals chaque pouvoir est seulement compétent d'une mantère exclusive pour un ou plusleurs aspects de cette matièren (R. Senelle et E. Van de Velde, La réforme de l'Etat belge, tome 111 , 1981, p. 25). "I I n'y a pas deux sortes de compétences réglonales ou communautaires : parce que lour définitlon repose sur l'ldée d'un partage d'attributlons, sans établissement de compétences concurrentes, toutes sont en principe exclusives" (J.M. Van Bol, "Les matlères communautalres et réglonales", Journal des Tribunaux, 1981, p. 637). "Les compétences de l'Etat, de la Communauté ou de la Région ont un caractère excluslf. Les autorltés de l'Etat, celles de la communauté ou celles de la Région peuvent seules agir dans les matlères qul sont respectivement de leurs attrlbutions" (C. Huberlant, Quelques aspects jur idiques des réformes Institutlonnel les, Liber aml car um Frederlc Dumont, 1984, p. 884). 
Alors que l'Etat, les Communautés et les Régions sont souvent présentés comme trois pouvoirs mis sur un pied d'égalité, l'expérience montre qu'il n'y a pas d'égalité réelle entre l'Etat, d'une part, et les Communautés ou les Régions d'autre part. A certains points de vue, il n'y a pas davantage d'égalité entre les Communautés et les Régions. Alors que les compétences nationales, communautaires et régionales sont souvent présentées comme des compétences exclusives, l'expérience montre que les Communautés et les Régions ne disposent guère de compétences réellement exclusives.

Au moment où la finalité et les implications de la réforme de l'Etat font à nouveau l'objet de controverses, il a paru intéressant de dresser, grâce à une série d'exemples concrets observés au cours des dernières années, l'inventaire des mécanismes juridiques engendrant la subordination de fait des Communautés et des Régions.

\section{Compétence et pouvoir}

Afin de comprendre les superpositions de compétences créées par la réforme de l'Etat, il convient de revenir un instant sur les notions de compétence et de pouvoir. Les compétences d'une institution, ce sont les matières dans lesquelles elle peut intervenir: la recherche, le tourisme, les travaux publics,... Les pouvoirs d'une institution, ce sont les moyens dont elle dispose pour exercer ses compétences : prendre des normes ayant valeur législative, adopter des règlements, percevoir des impôts, effectuer des dépenses, accomplir des actes administratifs, participer à la création de normes internationales (5).

Dans chaque compétence peuvent dont être exercés différents pouvoirs. En matière de sidérurgie, par exemple, domaine qui relève indiscutablement de la compétence législative de l'Etat dans le cadre de la loi spéciale du 8 aout 1980, l'Etat peut effectuer plusieurs actes. II peut adopter des lois (comme la loi du 5 mars 1984 relative au financement des secteurs nationaux) élaborer des règlements (comme les arrêtés d'exécution de la loi du 5 mars 1984), effectuer des dépenses (comme

(5) Pour une analyse succincte de cette problématlque dans le domalne des extensions de compétences du pouvolr exécutlf, volr F. Dehousse, "Pouvolrs spéclaux et changement de réglme (11) : l'insertion des pouvolrs spéclaux dans l'évolution du réglme parlementaire belge", Journal des Trlbunaux, 1985, pp. $65-72$ et $81-90, n^{\circ} 30$. 
celles effectuées par le truchement de la S.N.S.N.), prendre des actes administratifs (répartir des quotas de production). Il peut également imposer des taxes (dans ce domaine spécifique, toutefois, une partie du pouvoir fiscal est exercée par la Communauté européenne du Charbon et de I'Acier) ou participer à la création de normes internationales (notamment les normes européennes relatives aux aides financières des pouvoirs publics).

Il existe plusieurs répartitions de compétences, parce qu'il existe en fait plusieurs types de compétences : législative, réglementaire, budgétaire, administrative, fiscale ou internationale. Les auteurs de la loi spéciale du 8 aout 1980 l'ont sans nul doute perçu (6). $0 \mathrm{r}$, si les compétences réglementaires, budgétaires et administratives paraissent, de manière générale, être subordonnées aux compétences législatives dans le cadre de la réforme de l'Etat, on ne peut en dire autant des compétences internationales et fiscales.

Ces dichotomies portent en fait atteinte à l'autonomie des Communautés et des Régions. La primauté de l'Etat dans les relations internationales lui permet d'intervenir dans les compétences communautaires et régionales. Sa suprématie dans le domaine fiscal aboutit au même résultat. D'autres facteurs produisent d'ailleurs des conséquences identiques : les transferts budgétaires conditionnels, la superposition des règles de compétence et la formulation de certaines règles de compétence. Tous ces éléments mettent en évidence l'absence d'une vision structurée de la réforme de l'Etat.

(6) On remarquera, à titre d'exemple, la manière dont l'exposé des motifs de la lol spéclale du 8 août 1980 définlt les compétences réglonales en matière de recherche scientlfique appllquée : "la Réglon dispose, en matlère de recherche scientlfique appllquée, pour les matlères pour lesquelles elle est exclusivement compétente, tant de la compétence normative que de la compétence exécutive et de la compétence d'affectatlon budgétalre" (Doc. Parl., Sénat, sess. $1979-1980, n^{\circ} 434 / 1$, p. 351 . 


\section{Ie contrôle des relations internationales}

Dans le cadre de la réforme de l'Etat, les Communautés et les Régions disposent de compétences législatives mais l'Etat contrôle largement les négociations internationales sur ces matières. Selon l'article 68 de la Constitution, "le Roi... fait les traités de paix, d'alliance et de commerce". L'article 59bis de la Constitution, complété par l'article 16 de la loi spéciale du 8 aout 1980, prévoit que les Conseils de communauté doivent donner leur assentiment aux accords internationaux dans les matières relevant de leurs compétences. L'article 81 de la loi spéciale prévoit l'association des exécutifs communautaires ou régionaux à la négociation des accords internationaux dans les matières communautaires ou régionales.

Ainsi, en vertu de l'article $4,6^{\circ}$ de la loi spéciale du 8 aout 1980, "la radiodiffusion et la télévision" relèvent en principe des Communautés. Néanmoins, l'Etat négocie toujours les accords internationaux sur ces matières. La nécessité d'une répartition internationale des fréquences radiophoniques a par exemple permis à l'Etat de répartir les fréquences d'émission et de définir les conditions de leur utilisation (7). L'actualité récente l'a encore montré. En janvier 1986, la reconnaissance de nombreuses "radios libres" par l'Exécutif de la Communauté française s'est heurtée aux objections du secrétaire d'Etat chargé des P.T.T., qui a évoqué "les répercussions que ces décisions peuvent avoir sur les plans de fréquence mis au point en France, en Allemagne et aux PaysBas, pays avec lesquels les négociations sont fort difficiles" (8). Pour des raisons semblables, il existe toujours un commissariat au tourisme au sein du ministère national des Communications alors que le tourisme relève en principe des

(7) Volr la lol du 30 julllet 1979 relative aux radiocommunications (Moniteur belge, 19 septembre 1979) et I'A.R. du 20 août 1981 réglementant l'établlssement et le tonctionnement des stations de radiodiffusion sonore locale (Moniteur belge, 19 septembre 1981 ).

(8) La Lanterne, 20 janvier 1986. 
De même, selon l'article $6, \S$ ler, VI, $4^{\circ}$, de la loi spéciale, "l'aide aux entreprises ou unités d'exploitation" relève, malgré certaines exceptions importantes, des Régions. Néanmoins, l'Etat négocie toujours les accords internationaux sur ces matières, notamment dans le cadre de la Communauté européenne.

La négociation par l'Etat de directives européennes relatives à la protection de l'environnement influe sur l'action des Régions en matière de politique de l'eau. La négociation de normes européennes relatives au droit des aides conditionne l'action des Régions en matière d'expansion économique ou d'initiative industrielle publique.

Néanmoins, il existe une différence essentielle entre les Communautés et les Régions. Les Conseils de communauté donnent leur assentiment aux accords internationaux portant sur les matières communautaires. Cela peut parfois poser un problème technique, car la complexité des règles de compétence engendre des incertitudes quant à la nécessité de soumettre certains accords internationaux aux Conseils. Cette exigence constitue néanmoins une garantie importante, susceptible d'inciter l'Etat à une concertation réelle. Rien de semblable, par. contre, n'existe au sujet des accords internationaux portant sur. les matières régionales. Ainsi, l'Etat négocie tout à fait librement des accords limitant l'action des Régions dans le domaine des aides aux entreprises, de la politique énergétique ou de la protection de l'environnement. Certaines matières relèvent ainsi à la fois des compétences normatives des Régions et des compétences internationales de l'Etat. Peut-on véritablement parler, dans pareils cas, de compétences exclusives?

On pourrait croire que, de toute façon, les accords internationaux conclus par l'Etat dans les matières régionales ont peu d'importance réelle tant qu'ils n'ont pas fait l'objet de mesures d'exécution prises par les institutions régionales. Cette objection fait peu de cas du caractère directement applicable de nombreuses normes internationales, et tout

(9) Sur ce polnt, volr F. Dehousse, "Les confllts budgétalres ...", op. clt., pp. 26-28. 
spécialement des normes européennes (10). A partir du moment où une norme internationale a été rédigée en des termes suffisamment précis pour ne pas nécessiter de mesures d'exécution, les institutions régionales n'ont plus à intervenir. En plus, dans la sphère européenne, la Cour de justice des Communautés européennes a adopté une conception extrêmement large des normes directement applicables (11). Par surcroit, en Belgique, tout accord international directement applicable bénéficie, dès l'aboutissement du processus requis par la Constitution, de la primauté tant vis-à-vis de la loi que du décret (12).

Dans cette matière, il convient de noter que I'exigence d'un assentiment des Conseils de communauté aux accords internationaux portant sur des matières communautaires pourrait un jour poser des problèmes au regard du droit européen. Pour le moment, les normes européennes ne concernent guère les matières communautaires. Cependant, la situation parait susceptible de modifier, comme l'indiquent les premières réunions du Conseil des ministres de la Culture, ainsi que les premières interventions de la Commission dans le domaine de la télévision et du tourisme.

Si jamais l'utilisation des règlements communautaires devait s'étendre à ces matières, I'assentiment des Conseils de communauté se heurterait à l'article 189 CEE. Cet article prévoit en effet que les règlements deviennent directement applicables dans tous les Etats membres sans requérir aucune mesure de réception dans l'ordre juridique interne. Un problème similaire pourrait se poser au sujet des directives dont la Cour de justice des Communautés européennes aurait reconnu le caractére directement applicable. En réalité, les Conseils de communauté n'auront pas à donner leur assentiment à d'éventuelles normes européennes relatives à l'audiovisuel ou au tourisme. L'intégration européenne aura ainsi pour effet d'accentuer le poids de l'Etat national dans les matières

(10) Sur le concept d'applicabllité directe, volr J. Verhoeven, "La notlon d'applicabllité directe du drolt international", Revue belge de drolt international, 1980, pp. 243-264 et réf. citées.

(11) Sur la doctrine de l'applicablilté directe en drolt européen, volr J. Megret, M. Waelbroeck, J.V. Louis, D. Vignes et J.L. Dewost, Le drolt de la Communauté économlque européenne, volume 10 : la cour de justice - les actes des institutions, tome 1, 1983, pp. 519-525; R. Jollet, Le droit institutionnel des Communautés européennes, tome 11, 1983, pp. 303-326.

(12) Voir le célèbre arrêt Le Skl de la Cour de cassation (Cass., 27 mal 1971, Pas., 1971, I, 919). 
communautaires (13).

Le contrôle des relations internationales par l'Etat établit en réalité une sorte de tutelle nationale sur les activités des Communautés, incitées à tout le moins au compromis, et surtout sur les activités des Régions. Dans bien des cas, ces dernières exercent davantage un rôle d'exécution qu'un rôle d'initiative, contrairement à ce que la définition formelle de leurs compétences pourrait laisser croire. Dans les années à venir, l'extension prévisible des matières sujettes aux réglementations internationales risque même de déboucher, de manière inattendue, sur un rétrécissement substantiel de l'autonomie des Communautés et des Régions.

(13) Pour avolr un aperçu des problèmes que cela pourrait poser à l'avenir, voir la communication de la commission au Consell des ministres relative à l'action communautalre dans le domalne du tourlsme (Bulletin des Communautés européennes, 1986, supplément 4/86) et la récente proposition de directlve de la Commission au sujet de la radiodiffusion (Bulletin des Communautés européennes, 1986, supplément 5/86). 
L'article 110, $\S 2$ de la Constitution attribue aux Communautés et aux Régions un pouvoir fiscal de principe mais prévoit que "la loi détermine...les exceptions dont la nécessité est démontrée". Il établit ainsi une tutelle de l'Etat sur les impôts communautaires et régionaux.

"Les Régions et les Communautés peuvent donc parâtre moins assurées de leur pouvoirs fiscaux que de leurs compétences matérielles. En effet, le législateur national conserve en matière fiscale une mission de surveillance généralisée, un pouvoir de tutelle législative" (J.C. Scholsem, "L'impact de la réforme des institutions sur les finances publiques et la fiscalité", Ann. dr., 1981, p. 251). La section de législation du Conseil d'Etat donne un sens très large à ce pouvoir de tutelle. Selon le Conseil d'Etat, "plusieurs auteurs sont d'avis que sur la base des dispositions actuellement en vigueur, les Communautés et les Régions ne peuvent pas établir un impôt sur une matière déjà soumise à un impôt de l'Etat" (Doc. Parl., C.C.F., sess. 1981-1982, no 13/2, p. 6). Cette interprétation des limites posées au pouvoir fiscal des Communautés et des Régions parâ̂t exagérément restrictive. Elle ne correspond pas, en outre, au commentaire de certains auteurs cités par le Conseil d'Etat à l'appui de sa thèse. L'article $110, \S 2$, de la Constitution peut parfaitement signifier que les Communautés et les Régions disposent du pouvoir d'imposer n'importe quelle matière, quitte à ce que l'Etat exerce par la suite sa tutelle fiscale.

Dans la réalité, les Communautés et les Régions n'ont guère d'autonomie fiscale. Même si la loi ordinaire de réformes institutionnelles du 9 aout 1980 les autorise en tout cas à modifier certains éléments des impôts dont le produit leur a été totalement ristourné par l'Etat, cette possibilité reste soumise à une éventuelle modification législative votée à la majorité simple. Cela a été mis en évidence par la loi du 5 mars 1984, qui a retiré les droits de succession des impôts ristournés aux Régions. A ces contraintes juridiques s'ajoute une contrainte sociologique essentielle. La pression fiscale conjuguée de l'Etat, des provinces et des communes atteint une 
intensité suffisante pour que toute initiative des Communautés ou des Régions risque de se heurter à des résistances psychologiques sérieuses (14).

Aussi, si les Communautés et les Régions disposent de compétences législatives, le pouvoir fiscal, dans ces matières, demeure très largement aux mains de l'Etat. Par exemple, en vertu de l'article 4, 30, de la loi spéciale du 8 aoút 1980, les beaux-arts relèvent des Communautés mais la fiscalité applicable à cette matière relève en fait, pour les raisons déjà évoquées, de l'Etat. De même, en vertu de l'article $6, \S$ ler, II, $1^{\circ}$, de la loi spéciale du 8 aout 1980, la protection de l'environnement relève en principe des Régions mais la fiscalité applicablè à cette matière relève, dans le contexte actuel, de l'Etat. Bien súr, dans certaines matières, comme la télévision ou le logement, les Communautés et les Régions peuvent éventuellement, on l'a vu, recevoir une compétence fiscale par le biais des impôts ristournés. Cette compétence demeure néanmoins précaire parce qu'elle reste soumise à une suppression éventuelle par une loi à majorité simple.

Le constat vaut encore davantage pour les compétences régionales que pour les compétences communautaires. En effet, les premières portent souvent sur des matières de nature économique. Ainsi, en vertu de l'article 6 , $\S$ ler, VI, $4^{\circ}$, de la $10 i$ spéciale du 8 août 1980, I'expansion économique régionale, et notamment 1 'aide aux entreprises $n$ 'appartenant pas aux secteurs nationaux, relève des Régions. Ces dernières attribuent donc divers types de subsides et d'avantages. Cependant, l'Etat central peut parvenir à un résultat identique en modulant, par exemple, les règles relatives à l'impôt des sociétés ou les taux de la taxe sur la valeur ajoutée.

Dans la réalité, il n'y a pas une grande différence entre un subside accordé par les Communautés ou les Régions à des conditions déterminées et une réduction d'impôt, une dépense fiscale, accordée par l'Etat aux mêmes conditions. Cette problématique a été abordée par le Conseil d'Etat dans un avis du 12 novembre 1980 portant sur un projet de subside à la construction et à la rénovation d'habitations. Ce projet, voté par le Sénat et la Chambre, est devenu la loi du 24 décembre 1980 (Moniteur belge du 31 décembre 1980).

(14) Volr néanmoins les décrets du Consell réglonal wallon du 29 juin 1985 relatifs à la taxe sur les jeux et parls (Moniteur belge, 6 septembre 1985)

et du 7 octobre 1985 sur la protection des eaux de surface (Monlteur belge, 10 Janvier 1986). 
Le projet de loi instaurait "un subside égal à dix pour cent des deux tiers de la base de perception sur laquelle la TVA a été acquittée par un particulier qui fait construire une nouvelle habitation destinée à-être occupée par lui-même ou qui fait procéder à des travaux de rénovation à une habitation existante, occupée ou non par lui-même". Le Conseil d'Etat considéra que ce subside paraissait, à première vue, relever des compétences régionales. Cependant, il le qualifia en fin de compte de "système de remboursement partiel, dans des cas bien déterminés, de la TVA due et acquittée par le mâ̂tre de l'ouvrage pour la construction et la rénovation d'une habitation". Le Conseil d'Etat se rangeait ainsi à la position du gouvernement, pour lequel il s'agissait "d'une restitution d'impôt sous la forme d'une subvention".

"Tant selon son intitulé et son texte même que selon l'exposé des motifs qui le précède, le projet de loi tend à établir un régime de subventions destiné à promouvoir la construction et la rénovation d'habitations. On pourrait dès lors ête tenté, à première vue, d'affirmer qu'en vertu de l'article 6, par. ler, IV, et, dans une mesure moindre, de l'article 6, par. ler, 40, de la loi spéciale du 8 aoút $1980 .$. , la matière réglée par le projet relève de la compétence des Régions. En effet, d'après les travaux préparatoires de la loi précitée, le terme 'logement' doit être pris dans un sens très large et couvre l'ensemble de la politique du logement, en ce compris, dès lors, l'octroi de subventions en vue de stimuler la construction d'habitations.

Cette thèse ne résiste toutefois pas à une analyse plus poussée. (...) Il ressort de la plupart de ses articles que sa portée réelle est d'ordre fiscal national et que son objet véritable est d'organiser un système de remboursement partiel, dans des cas bien déterminés, de la TVA due et acquittée par le mâtre de l'ouvrage pour la construction ou la rénovation d'une habitation. (...) D'une part, la provenance des fonds destinés aux 'subsides', à savoir un prélèvement opéré sur les recettes de la TVA maintenues séparées et, d'autre part, le mode de détermination du montant de ces 'subsides', à savoir un pourcentage de la TVA acquittée, montrent que les prétendus subsides constituent de toute évidence une ristourne partielle de la TVA payée $(. .$.$) .$

Le fait que l'exécution administrative du système projeté soit confiée au ministère des Finances, loin d'attester que ce ministère jouera exceptionnellement et sans doute pour la première fois le rôle de pouvoir subsidiant, constitue au contraire une indication supplémentaire révélatrice de la nature fiscale de ce système" (Doc. Parl., Sénat, sess. 1980- 
$\left.1981, n^{0} 535 / 1, \mathrm{pp} .34-35\right)$.

Cette analyse semble erronée dans la mesure où un subside calculé en fonction de paramètres servant à établir une taxe ne peut être confondu avec une réduction d'impôt, même lorsque la taxe en question sert à son financement. Au cours des débats parlementaires, le ministre des Finances a lui-même reconnu qu'il ne s'agissait pas d'un véritable remboursement.

"La sixième directive du Conseil des Communautés européennes, relative à l'harmonisation des législations des Etats membres en matière de taxe sur le chiffre d'affaires, rend impossible la stimulation de la construction d'habitations, soit par une réduction directe du taux de la TVA, soit par un vrai remboursement de la TVA au profit du maitre de l'ouvrage" (Doc. Parl., Sénat, sess. 1980-1981, n 535/2, p. 2).

Une restitution d'impôt ne peut être confondue avec une réduction ou une exemption d'impôt. Admettrait-on, par exemple, qu'un subside aux entreprises calculé au moyen de données servant à établir l'impôt des sociétés relève de l'Etat? Ou qu'un subside aux familles calculé au moyen de données servant à l'impôt des personnes physiques relève de l'Etat ? Cette démarche aboutirait à vider les compétences communautaires et régionales de leur signification. Tout subside constitue, au fond des choses, une restitution d'impôt plus ou moins directe aux bénéficiaires (15).

Il reste que, si l'Etat avait agi par la voie d'une exemption d'impôt ou d'une réduction du taux de la TVA, ce qui se fait actuellement, il aurait valablement exercé dans le domaine du logement une influence importante, plus importante sans doute que l'influence des institutions régionales. Si le gouvernement n'a pas agi ainsi, c'est parce qu'il estimait à l'époque que les normes européennes relatives à la TVA l'en empêchaient.

(15) "Ne faut-11 pas ... considérer en matlère de subventions que la llgne de partage des compétences entre la lol et le décret se sltue allleurs, sans référence à la technique fiscale? Au vu du mode de répartition des compétences adopté par le constituant et la lol spéclale, c'est l'objectlf de la mesure envisagée qui paraft s'imposer comme premler crltère : dans quelle politique la subvention en projet s'inscrit-elle ? (...) La subvention sera donc à régler par décret ... lorsqu'elle s'inscrit dans le cadre des compétences des Communautés ou des Réglons; elle relèvera du pouvolr national dans les autres cas" (J.M. Van Bol, Deux années d'application, Les compétences réglonales et communautalres, 1983, p. 107). 
Le problème a été abordé une deuxième fois par le Conseil d'Etat, dans un contexte différent, lorsqu'il a été consulté sur le texte initial de la loi du 31 juillet 1984 (Moniteur belge du 10 août 1984). Dans une série de dispositions relatives à la "promotion de la reconversion industrielle", le gouvernement avait en effet mêlé des textes relatifs à certaines immunités fiscales destinées aux investisseurs et à l'attribution d'aides publiques. Le Conseil d'Etat a considéré que les mesures fiscales ne justifiaient pas l'introduction d'une réglementation nationale dans le domaine de l'expansion économique régionale.

"La création d'un cadre en matière de financement des initiatives dans le domaine de l'expansion économique régionale a été en elle-même exclue des compétences nationales par l'attribution d'une compétence exclusive aux Régions pour I'expansion économique régionale" (Doc. Parl., Chambre, sess. 19831984, $n^{0} 927 / 1$, annexe, p. 18).

On a généralement considéré que les dispositions incriminées relevaient plutôt de l'initiative industrielle publique que de l'expansion économique. Cela n'ote rien, cependant, au bienfondé de l'objection de principe du Conseil d'Etat : l'Etat ne peut invoquer son pouvoir fiscal pour justifier l'adoption de normes nationales dans les matières communautaires ou régionales.

Toutefois, même si l'Etat ne peut utiliser sa suprématie fiscale afin d'instaurer des réglementations ou des subsides dans les matières communautaires et régionales, cela ne diminue guère ses facultés d'intervention dans lesdites matières. La dichotomie introduite dans le cadre de la réforme de l'Etat entre le pouvoir législatif et le pouvoir fiscal engendre une conséquence essentielle. A partir du moment où une matière peut se situer à la fois dans les compétences fiscales de l'Etat et dans les compétences législatives des Communautés ou des Régions, le rapport d'importance entre l'Etat, les Communautés et les Régions dépend de manière directe, et avant tout, de la capacité financière respective de ces trois institutions. Cela explique l'énorme intérêt suscité par les moyens financiers des Communautés et des Régions au cours des dernières années, plus encore que par leurs compétences normatives. 
Dans le domaine budgétaire, la réforme de l'Etat de 1980 a défini des règles nettement moins précises que dans le domaine international ou le domaine fiscal. Pour cette raison, les problèmes de compétence relatifs à l'exercice du pouvoir budgétaire, c'est-à-dire au pouvoir d'effectuer des dépenses, ont été particulièrement nombreux au cours des dernières années. Suite à de multiples controverses, le principe de la subordination des dépenses de l'Etat, des Communautés et des Régions à leurs compétences normatives respectives a finalement été consacré. Néanmoins, ce principe n'a pas toujours été respecté, notamment dans le cadre des interventions du Fonds de rénovation industrielle ou des "invests" (16).

Cela dit, il importe de réaliser que, d'un point de vue concret, I'exclusivité des compétences budgétaires des Communautés et des Régions se trouve hypothéquée par leur dépendance financière vis-à-vis de l'Etat. Non seulement ce dernier dispose d'une suprématie fiscale, mais il a la possibilité de modifier les dotations attribuées aux Communautés et aux Régions. Ces dotations ont été définies, comme les impôts ristournables, dans la loi ordinaire de réformes institutionnelles du 9 aout 1980. Elles peuvent, par conséquent, être modifiées par un vote à la majorité simple.

En mai 1986, dans le cadre du plan d'économies de ValDuchesse, le gouvernement a prévu une réduction des dotations communautaires et régionales de FB 6,6 milliards. Les différents exécutifs concernés ont protesté contre la décision. A cette occasion, le président de l'Exécutif régional wallon a déclaré que l'Etat modifiait "un instrument de base de la régionalisation, en changeant unilatéralement un contrat" (17).

(16) Sur ce polnt, volr F. Dehousse, "Les conflits budgétalres ...", op. cit., pp. 7-31.

(17) La Libre Belgique, 21 juln 1986. 
La remarque a un fondement politique ou sociologique. Cependant, elle n'a pas de fondement juridique. Pour qu'une disposition ait vraiment valeur de contrat entre les deux grandes communautés du pays, elle doit être inscrite dans un texte dont la modification requiert l'approbation d'une majorité dans chaque communauté. Ce n'est pas le cas des dispositions financières de la réforme de l'Etat. Le président de l'Exécutif régional wallon l'a d'ailleurs reconnu par la suite : "sur le plan financier, c'est l'organisation de la dépendance...parce que les finances régionales sont réglées par une loi à majorité simple" (17).

On peut s'interroger sur la justification de la dichotomie établie dans la réforme de l'Etat entre les compétences matérielles des Communautés et des Régions, définies dans une loi à majorité spéciale, et leurs moyens financiers, définis dans une loi à la majorité simple. En effet, il existe un lien tellement étroit entre compétence et dépense dans les Etats contemporains que ce système semble tout à fait artificiel. Avant 1980, le Conseil d'Etat paraissait favorable à cette dichotomie; par la suite, il a davantage souligné les rapports entre les compétences et les ressources financières (18).

Comme l'ont montré les dernières années, cette situation aboutit en tout cas à un rabaissement de la position des Communautés et des Régions (19). Ce rabaissement se trouve encore accentué par. les charges financières supplémentaires que l'Etat a imposées aux institutions communautaires et régionales dans la loi du 5 mars 1984 relative aux charges du passé.

La loi du 5 mars 1984 (Moniteur belge du 16 mars 1984) concerne à la fois le financement des secteurs nationaux et les

(17) volr p.17.

(18) "La lol ordlnalre du 9 août 1980 est à ce polnt importante pour l'exerclce des compétences transférées aux Communautés et aux Réglons par la lol spéclale du 8 août 1980 ..." (Doc. Parl., Chambre, sess. 1983-1984, n834/1, p. 27).

(19) La falblesse des moyens financlers des Communautés et des Réglons au regard de leurs compétences matérlelles risque, en outre, de susciter la tentation de mesurer les compétences à l'aune des moyens par soucl de cohérence. La Cour d'arbltrage semble avoir cédé quelque peu à cette tentation dans son arrêt du 25 févrler 1986 relatif à la Calsse natlonale de crédlt professionnel (sur cet arrêt, volr $F$. Dehousse, "Une première approche du rôle de l'Etat et des Réglons dans le domalne de la politlque économique", Journal des Tribunaux, 1986, pp. 611-616). 
charges du passé. Elle contraint notamment les Régions à rembourser les emprunts relatifs à certaines matières régionales, essentiellement le logement, et contractés entre 1975 et 1980. Comme les Régions ne possédaient pas une personnalité juridique à l'époque et que les exécutifs régionaux siégeaient au sein du gouvernement national, le principe de cette imputation de dettes rétroactive pourrait être contesté. On pourrait éventuellement considérer qu'elle viole l'article 95 de la loi spéciale du 8 aout 1980, qui prévoit l'entrée en vigueur de la loi spéciale le ler octobre 1980. Le procédé vise en tout cas à réduire les moyens financiers des Régions sans devoir passer par une modification formelle des dotations régionales et des impôts ristournés (20).

Le traitement différencié des compétences matérielles et des moyens dans la réforme de l'Etat se double d'une inégalité au point de vue financier, entre les Communautés et les Régions. Les Régions ont reçu des moyens nettement moins importants que les Communautés. Or, les compétences régionales ne présentent certainement pas un caractère moins onéreux que les compétences communautaires. En période de crise, les responsabilités économiques des Régions amènent plutôt à la conclusion inverse. Le résultat de cette autre dichotomie n'a pas tardé : depuis l'entrée en vigueur de la réforme de l'Etat, les politiques régionales ont du subir des restrictions beaucoup plus amples que les politiques communautaires.

Cette inégalité a encore été accentuée par la loi du 5 mars 1984, qui impute des dettes antérieures à l'entrée en vigueur de la loi spéciale du 8 aout 1980 aux seules Régions, et non aux Communautés. Ces dettes représentent une charge considérable pour les budgets régionaux. Comme le notait la presse, "pour la Wallonie, par exemple, la charge prévue pour 1985 équivaut, à 6 milliards près, à l'ensemble de ses ressources annuelles" (21).

La mise sous tutelle financière des Communautés et des Régions éveille la tentation d'un mécanisme hybride : le transfert budgétaire conditionnel. Ainsi, au cours des dernières années, des ressources additionnelles ont été transférées à plusieurs reprises aux Régions : crédits de recherche répartis selon des

(20) Sur la lol du 5 mars 1984, volr J.C. Scholsem, "Aspects juridiques de la lol du 5 mars 1984 relative aux soldes et aux charges du passé et aux secteurs économlques nationaux", Bull. doc. mln. fin., septembre 1985, pp. 130149; F. Dehousse, "Les conflits budgétalres...", op. cit., pp. 48-68.

(21) Le Solr, 8 novembre 1984. 
clefs de répartition complexes, moyens supplémentaires accordés par le Fonds de rénovation industrielle aux sociétés régionales d'investissement. L'Etat intervient dans la gestion de certaines de ces ressources. Par exemple, il participe, par le biais du Fonds de rénovation industrielle, aux décisions économiques des sociétés régionales d'investissement (22).

Plus récemment; la perspective d'un transfert de ressources aux Communautés en vue de financer l'exercice de compétences additionnelles en matière d'enseignement a été évoquée. Les Communautés, dans cette perspective, géreraient l'enseignement sans révision de l'article 59bis de la Constitution, en fonction de normes définies par l'Etat (23). De pareilles constructions établissent un système de compétences superposées et un contrôle de l'Etat sur certaines activités des Communautés et des Régions. De ce fait, elles vont à l'encontre des principes essentiels de la réforme de l'Etat.

(22) Sur les problèmes posés par le fonds de rénovation industrielle, voir "Le Fonds de rénovation industrlelle", Courrler Hebdomadalre du CRISP, $n^{\circ} 1115-1116,25$ avrll 1986; F. Dehousse, "Les confllts budgétalres ...", op. clt., pp. 44-47.

(23) Sur les perspectives de communautarlsation de l'enselgnement, volr $C$. Simon, "La communautarlsation de l'enselgnement", Courrler Hebdomadalre du CRISP, $n^{\circ} 1121,13$ juin 1986; F. Dehousse, "Les conflits budgétalres...", op. clt., pp. 44-47. 


\section{La construction des règles de compétence}

Il existe, en outre, plusieurs techniques de rédaction des règles de compétence qui tendent à restreindre, dans les faits, l'autonomie des Communautés et des Régions, ainsi que l'exclusivité de leurs compétences.

Une première technique consiste à réserver, dans de nombreușes matières, la législation organique à l'Etat. Elle a surtout été utilisée dans le cadre des compétences communautaires. La restriction peut être exprimée de façon explicite. Ainsi, l'article 5, § ler, I, de la loi spéciale du 8 aout 1980 réserve à l'Etat "la législation organique" et "les règles de base" en matière de soins de santé. L'article $5, \S 1$, II, lui réserve les "règles organiques" des centres publi.cs d'aide sociale en matière d'aide aux personnes.

La restriction peut aussi être exprimée de façon implicite. Ainsi, I'article 59bis, $\S 2,2^{\circ}$, de la Constitution attribue aux Communautés une compétence de principe dans le domaine de l'enseignement; néanmoins, il réserve à l'Etat toutes les questions essentielles concernant l'organisation de l'enseignement. La compétence des Communautés se résume, en fín de compte, à peu de choses. De même, l'article $5, \S l e r$, II, $6^{\circ}$, de la loi spéciale du 8 aoút 1980 attribue aux Communautés "la protection de la jeunesse à l'exception des matières relevant du droit civil, du droit pénal ou du droit judiciaire".

En fait, cela revient à limiter les compétences communautaires à des compétences accessoires, qui ne peuvent en rien modifier l'application des lois organiques. Le phénomène a été mis en évidence par la Cour d'arbitrage dans son arrêt du 22 janvier 1986 relatif à la matière de l'enseignement. Cet arrêt annule le décret du Conseil de la Communauté française du 2 décembre 1982, qui organisait la subsidiation d'établissements d'enseignement fondamental sur le territoire de la région flamande 
et à l'extérieur de la Belgique (24).

A I'origine, le texte devait s'appliquer à tous les types d'enseignement. Toutefois, l'incompétence des Communautés au sujet des diplômes obligea le Conseil de la Communauté française à réduire le champ d'application du décret à l'enseignement fondamental. Ceci montre combien, dans la réalité, les initiatives prises par les Communautés se heurtent rapidement à la barrière des compétences nationales, ne serait-ce qu'au sujet des diplômes, des structures de l'enseignement ou de l'obligation scolaire.

La contradiction plane d'ailleurs sur l'ensemble de l'arrêt du 22 janvier 1986. Ce dernier définit la compétence communautaire en matière d'enseignement d'une façon à première vue fort large : "chaque Communauté peut...créer de nouvelles sortes d'enseignement, de nouvelles orientations, de nouvelles méthodes et mettre sur pied des expériences". En affirmant que le décret communautaire "ne peut aller à l'encontre des principes fondamentaux établis par le législateur national", la Cour d'arbitrage laisse même entendre qu'un décret communautaire pourrait éventuellement porter sur des aspects secondaires de ces matières réservées. L'annulation du décret du 2 décembre 1982 prouve toutefois que "les principes fondamentaux" englobent bon nombre de détails et que l'autonomie de principe des Communautés se trouve en réalité singulièrement limitée.

Une autre technique de rédaction des règles de compétence, plus souvent utilisée dans le cadre des compétences régionales, aboutit au même résultat. Elle consiste à subordonner directement l'action des Communautés et des Régions au respect des normes nationales. Ainsi, l'article $6, \S l e r$, II, de la loi spéciale du 8 août 1980 subordonne la compétence régionale en matière de protection de l'environnement au respect des "normes légales". L'article 6, §ler, VI, 30, subordonne la compétence régionale en matière de politique du crédit aux "règles imposées dans le cadre des politiques nationales monétaires et du crédit". L'article $6, \S$ ler, VI, 40, subordonne la compétence régionale en matière d'expansion économique au "cadre normatif dans lequel s'exercent l'activité économique et la concurrence".

(24) Sur cet arrêt et les références des textes léglslatlfs en cause, voir F. Dehousse, "Note sous l'arrêt de la Cour d'arbltrage du 22 janvler 1986", Journal des Trlbunaux, 1986, pp. 496-499. 
Cela revient à instaurer un principe de primauté limitée des normes nationales sur les normes régionales. Lois et décrets ont une force juridique égale...pourvu que les décrets se coulent dans les limites définies par la loi.

Une troisième technique, surtout utilisée dans le cadre des compétences régionales, va plus loin. Elle consiste à n'attribuer aux Communautés et aux Régions que des compétences d'exécution. Ainsi, les Régions appliquent les lois d'expansion économique, en vertu de $1^{\prime}$ article $6, \bar{\S}$ ler, VI, $4^{\circ}$, de la loi spéciale du 8 août 1980, mais ces lois ne peuvent être modifiées que par l'Etat, avec l'accord des exécutifs régionaux. Les Régions exécutent, en vertu de l'article 6 , $\S$ ler, IX, $2^{\circ}$ et $3^{\circ}$, les normes nationales relatives au placement des chômeurs ou à l'occupation des travailleurs étrangers.

Cette technique nie l'autonomie des Communautés et des Régions, ainsi que l'exclusivité de leurs compétences. Bien sûr, les décisions prises par les institutions communautaires ou régionales en vertu des normes nationales ne font pas l'objet d'une tutelle. Néanmoins, elles peuvent être attaquées devant les juridictions, ce qui aboutit à une subordination.

I1 arrive même que la loi spéciale du 8 août 1980 définisse les compétences communautaires et régionales par référence à des lois ordinaires. Des compétences définies en principe à la majorité spéciale dépendent ainsi, en pratique, d'une loi à majorité simple. Ainsi, l'article 6, §ler, VII, b, définit la compétence régionale en matière de distribution publique du gaz par référence à "l'article ler de la loi du 2 avril 1965 relative au transport des produits gazeux". L'article 6 , $\S$ ler, VIII, $2^{\circ}$, définit la compétence régionale en matière de pouvoirs subordonnés par référence à la législation sur le fonds des communes. Que se passera-t-il si ces législations sont radicalement modifiées, voire abrogées? Rien ne le précise. La compétence régionale en matière de distribution de l'électricité dépend, selon l'article $6, \S 1 e r, V I I, a$, de la loi spéciale du 8 août 1980, des "règles de comptabilisation du Comité de contrôle de l'électricité et du gaz". De pareils procédés légistiques peuvent apparaître incompatibles avec la notion de loi à majorité spéciale.

La définition des compétences communautaires et régionales par. référence à des lois ordinaires ou à des normes encore moins formelles se prête évidemment à plusieurs interprétations. La référence peut signifier que les compétences en question doivent être interprétées au regard de l'état au ler octobre 1980 des textes auxquels on fait référence. Elle peut également signifier que l'Etat a la possibilité de changer, d'ac- 
croître ou de réduire les compétences en question par une simple modification de ces textes. Le procédé est, à tout le moins, ambigu.

A ces éléments, il convient d'ajouter le caractère restrictif des travaux préparatoires de la réforme de l'Etat. Alors que bon nombre des dispositions de la loi spéciale du 8 aout 1980 recourent à des termes généraux, les travaux préparatoires tendent le plus souvent à réduire leur portée. Cela constitue d'ailleurs une difficulté d'interprétation majeure, dans la mesure où ce procédé débouche parfois sur des contradictions entre les textes législatifs et les documents destinés à les éclairer. Il ne s'agit pas, à proprement parler, d'une technique de rédaction; la tendance a néanmoins des conséquences importantes.

Ces éléments montrent que les Communautés et les Régions disposent d'une autonomie moins grande qu'un examen superficiel des textes peut le laisser croire. Elles disposent d'institutions propres et d'un pouvoir décrétal, certes, mais leurs compétences on souvent été définies en termes de subordination.

La remarque s'applique d'ailleurs plus aux Régions qu'aux Communautés. Les compétences communautaires ont davantage été définies en blocs de matières, surtout dans les matières proprement culturelles énumérées par l'article 4 de la loi spéciale du 8 aout 1980. La subordination des normes communautaires aux normes nationales caractérise déjà plus les matières dites personnalisables, énumérées par l'article 5 de la loi spéciale. Toutefois, cette subordination pèse surtout sur: les compétences régionales. Cela explique d'ailleurs pourquoi les activités décrétales des Conseils de Communauté ont été beaucoup plus développées que celles des Conseils régionaux au cours des six dernières années. 
Un cinquième facteur est susceptible d'hypothéquer l'exclusivité des compétences communautaires et régionales : il s'agit de la superposition des règles de compétence. La loi spéciale du 8 août 1980 et l'article 59bis de la Constitution ont en effet instauré un enchevêtrement de frontières de compétence tout à fait extraordinaire. Le phénomène a déjà été souligné par bon nombre d'auteurs. Toutefois, les problèmes concrets apparus pendant les dernières années permettent de mieux mesurer ses implications.

La réforme de l'Etat a, tout d'abord, découpé certaines matières avec minutie. La politique du tourisme relève des Communautés, l'aide à l'industrie du tourisme des Régions, et 1 'accès aux professions touristiques de l'Etat. La radiodiffusion et la télévision relèvent de la Communauté, mais les communications du gouvernement national et la publicité commerciale relèvent de l'Etat. La loi organique des intercommunales relève de l'Etat, mais les modalités de fonctionnement, le contrôle et la fixation du ressort des intercommunales relèvent des Régions. La recherche fondamentale relève de l'Etat et la recherche appliquée des Régions. Les exemples abondent.

L'enseignement et la politique énergétique fournissent deux autres illustrations. Dans le domaine de l'enseignement, l'article 59bis, $\S 2,2^{\circ}$, de la Constitution distingue huit aspects différents : sept aspects essentiels relèvent de l'Etat et la compétence résiduaire appartient aux Communautés. Dans le domaine de l'énergie, l'article $6, \S 1 \mathrm{er}$, VII, de la loi spéciale du 8 aout 1980 distingue douze aspects différents : cinq de ces aspects relèvent de l'Etat et sept des Régions. Le législateur a précisé, en outre, que cette énumération ne devait pas être considérée comme exhaustive. Il arrive aussi que la loi attribue à deux pouvoirs distincts les aspects normatifs et les aspects budgétaires d'une même matière. Cette dichotomie a déjà été soulignée dans le domaine de l'enseignement ou du tourisme. Elle se manifeste dans d'autres 
secteurs. Ainsi, le cinéma, en tant que partie des beaux-arts, relève des Communautés alors que l'aide à l'industrie cinématographique relève de l'Etat. La politique familiale relève des Communautés et les allocations familiales de l'Etat. D'autres règles de compétence se cantonnent parfois à des attributions non définies, conférant par exemple aux Régions "les aspects régionaux" de certaines matières, sans autre précision.

Au découpage tatillon des différents aspects de nombreuses matières s'ajoutent deux autres problèmes. En premier lieu, les règles de compétence utilisent des critères variés : elles se réfèrent parfois à un objectif (l'utilisation rationnelle de l'énergie), à un cadre juridique (les modalités de fonctionnement des intercommunales), à une matière (la production de l'eau) ou à une législation spécifique (la législation organique sur la dispensation de soins). Cette multiplicité de critères favorise la superposition des règles de compétence.

En deuxième lieu, il faut noter que cette superposition se produirait de toute façon, même si les compétences de l'Etat, des Communautés et des Régions avaient été définies de manière plus simple. Une matière unique peut en effet se situer au carrefour de plusieurs compétences. Si les charbonnages relèvent de la politique économique, ils concernent également la politique de l'énergie et l'exploitation des ressources naturelles. Dans des secteurs relevant de l'expansion économique régionale, comme l'aéronautique ou les télécommunications, les commandes publiques jouent un rôle fondamental. Entre les subsides et les marchés publics existent des liens. De même, la politique des travaux publics entretient des rapports étroits avec l'aménagement du territoire. Bien souvent, la réalité est une, et sa division entre plusieurs matières constitue une (simple) frontière conceptuelle.

\section{Trois exemples concrets}

La matière du tourisme offre une première illustration des problèmes posés par la superposition des règles de compétence. L'article 4, $10^{\circ}$, de la loi spéciale du 8 aout 1980 range dans les compétences communautaires "le tourisme". L'article $6, \bar{\beta}$ ler, VI, 40, c, range dans les compétences régionales "I'aide à l'industrie du tourisme". Toutefois, en vertu de l'article 6 , $\S$ ler, VI, deuxième partie, $6^{\circ}$, les "conditions d'accès à la profession" continuent à relever de l'Etat.

Cette situation aboutit à une confusion. Les aides au tourisme figurent à la fois dans les budgets communautaires et régio- 
naux, ce qui constitue une source possible de gaspillage. Les Communautés éprouvent des difficultés à mener une politique touristique puisque l'ensemble du statut de la profession touristique relève de l'Etat. Par surcroît, ce dernier intervient encore dans la politique du tourisme à Bruxelles.

La Cour d'arbitrage est intervenue dans ce domaine. Suite à un recours en annulation intenté par l'Etat, elle a statué sur la validité du décret du Vlaamse Raad du 20 mars 1984 portant statut des centres d'hébergement. Dans son arrêt du 26 juin 1986, la Cour d'arbitrage a donné une interprétation extrêmement large à la compétence communautaire. En fait, elle n'a guère tenu compte des compétences nationales en matière de conditions d'accès à la profession, considérant que cette restriction ne concernait que l'action des Régions (25). L'interprétation présente l'avantage de simplifier la matière. Néanmoins, elle ne correspond pas aux dispositions légales.

La matière de l'énergie nucléaire of re une deuxième illustration des problèmes posés par la superposition des règles de compétence. L'article $6, \S$ ler, VII, deuxième partie, $c$, de la loi spéciale du 8 aout 1980 range dans les compétences nationales "le cycle du combustible nucléaire". L'article 6 , \$ ler, II, 30, range dans les compétences régionales "la police des établissements dangereux, insalubres et incommodes". Cette situation complique l'implantation des centrales nucléaires. L'Etat éprouve naturellement des difficultés à mener seul la politique nucléaire si les permis d'exploitation doivent être accordés par les Régions.

La section d'administration du Conseil d'Etat est intervenue dans ce domaine. Suite à un recours en annulation intenté par une association, elle a statué sur l'autorisation d'implantation de la deuxième centrale nucléaire de Tihange accordée par l'Etat. Dans son arrêt du 4 avril 1986, le Conseil d'Etat a donné une interprétation extrêmement large à la compétence nationale. En fait, il n'a guère tenu compte de la compétence régionale en matière de police des établissements dangereux, estimant que ce vocable n'englobait pas les autorisations

(25) "On ne peut appliquer aux Communautés l'exception de l'article 6, par. ler, VI, dernler alinéa, $6^{\circ}$ de la lol spéclale du 8 août 1980 qui vise expressément les Réglons. Dans la mesure où Il est établi que le Vlaamse Raad, en flxant le statut des entreprises d'hébergement, exerce une compétence conformément à ... la Constitution, ... Il est contraire à la Constitution et à la lol de recourir à une exception visant le seul exercice de compétences réglonales", Moniteur belge, 15 julllet 1986. 
d'implanter les centrales nucléaires (26). Ici aussi, l'interprétation présente l'avantage de simplifier la matière; néanmoins, elle ne correspond pas non. plus aux dispositions légales.

La matière des intercommunales fournit une troisième illustration des problèmes posés par la superposition des règles de compétence. L'article $6, \S$ ler, VIII, $1^{\circ}$, de la loi spéciale du 8 aout 1980 range dans les compétences régionales "les modalités de fonctionnement, le contrôle et la fixation du ressort des associations de communes dans un but d'utilité publique, ainsi que l'application des lois organiques relatives à ces associations". Cette disposition présente plusieurs ambiguités, encore accentuées par les contradictions des travaux préparatoires. Aussi, depuis l'entrée en vigueur de la réforme de l'Etat, elle a suscité des interprétations diverses.

De 1980 à 1983, la section de législation du Conseil d'Etat a rendu trois avis successifs sur le sujet. Après avoir hésité, elle a conclu que les Régions ne disposaient d'aucune compétence normative en matière d'intercommunales. Ultérieurement, suite à un recours en annulation de l'Etat contre le décret du Conseil régional wallon du ler février 1985, la Cour d'arbitrage est intervenue dans ce domaine. En annulant le décret contesté, elle a fourni une nouvelle interprétation de l'arti-

(26) Sur cet arrêt, voir F. Dehousse, "La réforme de l'Etat et l'énergle nucléalre", Ann. dr. Llège, 1986, pp. 338-346.

"La pollice des établlssements dangereux, insalubres et Incommodes n'a été attribuée à la compétence des Réglons par l'artlcle 6, par. ler, $11,3^{\circ}$, de la lol spéclale de réformes institutionnelles du 8 août 1980 que 'sous réserve des dispositions relatives à la protection du travall'. (...) Dès lors, relève seule de la compétence des Régions la pollce, dlte 'externe' des établissements classés. (...) sl le réglme des établissements classés possède un champ d'appllication en princlpe tout à falt général, Il n'a cependant jamals été étendu aux établissements présentant un danger de radlations ionisantes. (...) La lol du 29 mars 1958 (relative à la protection contre les radiations lonisantes) a implicitement, mais certainement, soustralt la police externe des établlssements détenant des substances capables d'émettre des radlations ionisantes au réglme des établissements classés du règlement général pour la protection du travall. (...) La matlère de la protection de la population contre les radiations lonlsantes est demeurée de la compétence de l'etat en raison de son appartenance à la politlque de l'énergle dans une matlère dont l'indivislbllité technlque et économlque requlert une mise en ceuvre homogène sur le plan national et de son llen évident avec "le cycle du combustible nucléaire'". 
cle $6, \S$ ler, VIII, $1^{0}$ de la loi spéciale du 8 août 1980 (27).

Suite à cet arrêt, la section de législation du Conseil d'Etat a modifié sa position lorsqu'elle a de nouveau été consultée sur un projet de loi relatif aux intercommunales. En tenant compte de l'intexprétation de la Cour d'arbitrage, elle a essayé d'effectuer entre l'Etat et les Régions un partage des dispositions organiques relatives aux intercommunales. Ainsi, selon ce partage, la forme juridique des intercommunales et la représentation des communes en leur sein constituent des "éléments essentiels" du statut des intercommunales; à ce titre, ils relèvent de l'Etat. Par contre, la détermination du siège des intercommunales constitue une modalité de fonctionnement et l'organisation de la tutelle sur les intercommunales une forme de contrôle; à ce titre, elles relèvent des Régions (28).

La dernière intexprétation du Conseil d'Etat confère un sens relativement étroit au vocable "modalités de fonctionnement". Néanmoins, elle correspond dans une large mesure aux dispositions légales. Cela se traduit, on le notera, par une plus grande complexité que dans les deux exemples précédents. Les compétences nationales et régionales s'interpénètrent à un point tel qu'on n'imagine pas l'adoption d'un statut complet des intercommunales sans une coordination des initiatives nationales et régionales.

Ces trois exemples montrent à quel point la réforme de l'Etat a établi une structure normative complexe. Cela n'étonnera guère les observateurs familiaxisés avec les systèmes fédéraux

(27) Sur ce conflit de compétence particullèrement complexe et les références qul s'y rapportent, voir $F$. Dehousse, "Un noeud de vipères constitutionnel : le statut des intercommunales et la Cour d'arbltrage", Journal des Trlbunaux, 1985, pp. 617-636.

"L'article 6, par. ler, VIII, $1^{\circ}$, de la lol spéclale opère donc un partage de compétence normative entre l'Etat et les Réglons :

a) la compétence normative des Réglons à l'égard des assoclations de communes est limitée aux modalités de fonctionnement, au contrôle et à la fixation du ressort des assoclations;

b) la compétence normative de l'Etat à l'égard des assoclations de communes comprend tout autre objet relatif à ces assoclations, et notamment les conditions d'affillation à une assoclation intercommunale et les condltions de retralt d'une telle assoclation".

(28) Doc. Parl. Chambre, sess. 1985-1986, $n^{\circ} 125 / 13$. Le Comité de concertation gouvernement-exécutifs a par la sulte adopté une position identlque (Doc. Parl., Chambre, sess. 1985-1986, n¹25/14). 
étrangers. Tous les Etats fédéraux, comme les Etats régionalisés, présentent une structure plus compliquée que les Etats unitaires. Cette complication peut trouver sa compensation, il faut le rappeler, dans un processus de décision plus efficace et plus proche du citoyen. Les exemples illustrent combien pareille structure impose une concertation étroite et permanente entre l'Etat, les Communautés et les Régions. Ainsi, la construction d'un pont requiert normalement une initiative de l'Etat, responsable des travaux publics, et une autorisation de la Région, responsable de l'urbanisme et de l'aménagement du territoire. De même, la réforme de l'audiovisuel requiert une initiative des Communautés, responsables de la politique audiovisuelle, et une intervention de l'Etat, responsable des normes relatives à la publicité commerciale. A cet égard, les dispositions de la loi spéciale du 8 août 1980 apparaissent insuffisantes, en dépit des quelques possibilités prévues. De plus, la concertation n'implique à l'heure actuelle que des échanges de vues. L'organisation d'un régime de conventions entre les pouvoirs et l'introduction d'actes conjoints constitueraient deux réformes utiles.

Cette carence de la loi spéciale du 8 aoút 1980 a été soulignée par le Conseil d'Etat dans son dernier avis sur les intercommunales. "L'article 6, par. 5, premier alinéa, de la loi spéciale du 8 août 1980 a prévu pour les associations de communes dont le ressort s'étend à plus d'une Région, des décisions prises, d'un commun accord entre les exécutifs concernés, mais ni la Constitution, ni la loi spéciale ne prévoient l'adoption de normes communes par deux Conseils régionaux".

Par ailleurs, les exemples repris témoignent de certaines complexités "gratuites" dans le cadre de la réforme de l'Etat. Ceci concerne la matière des intercommunales, où la réforme impose des distinctions subtiles en ce qui relève des dispositions organiques et ce qui relève des modalités de fonctionnement, du contrôle ou de la fixation du ressort des intercommunales. La même remarque vaut également pour d'autres matières. Dans le domaine des travaux subsidiés, la loi spéciale du 8 août 1980 attribue la réparation des trottoirs à l'Etat et la réparation des égouts aux Régions. Dans le domaine de la recherche, elle range la recherche fondamentale dans les compétences nationales et la recherche appliquée dans les compétences régionales (29).

(29) Sur ces exemples, voir F. Dehousse, "Les conflits budgétaires ...", op. cit., pp. 24-31. 
Toute restructuration d'un Etat moderne pose de nombreuses difficultés. A notre époque, l'Etat exerce tant de fonctions différentes que leur scission présente toujours un caractère compliqué. Cependant, la réforme de l'Etat belge va au-delà des complications nécessaires. Elle laisse à l'observateur le sentiment que le législateur n'a pas pu, à certains moments, prendre une décision et qu'il a entretenu la confusion. Cela s'explique par le fait que la nouvelle structure constitutionnelle de la Belgique n'a pu faire l'objet en 1980 d'un véritable accord politique. En outre, elle ne procède pas d'un modèle clair. D'où son ambiguité.

A l'examen, l'autonomie des Communautés et des Régions se révèle être un principe fort théorique. Certains mécanismes permettent en effet à l'Etat d'établir des formes de tutelle indirecte. La dichotomie entre compétences législatives et internationales instaure un système de compétences concurrentes, où l'intervention de l'Etat conditionne l'activité des Communautés et gouverne l'activité des Régions. La dichotomie entre compétences législatives et fiscales établit un système à géométrie variable, dans lequel l'intervention de l'Etat se superpose à l'action des Communautés ou des Régions. Ces dernières demeurent, en plus, dans une dépendance financière permanente.

L'exclusivité des compétences communautaires et régionales se révèle aussi être un principe théorique. Dans les faits, il n'existe pas tellement de compétences communautaires et régionales exclusives. Cela explique la difficulté de la tâche réservée tant à la Cour d'arbitrage qu'à la section de législation du Conseil d'Etat. Ces institutions doivent en permanence passer en revue un grand nombre de règles afin de vérifier si l'une ou l'autre d'entre elles fournit un fondement à telle ou telle activité de l'Etat, des Communautés ou des Régions. Elles doivent, en outre, prendre en considération l'existence, entre ces règles de compétence, d'une importante quantité de domaines mixtes. Il faut, au fond, identifier des compétences exclusives dans un système où le maintien d'une stricte exclusivité des compétences a précisément été rendu 
fort aléatoire.

Les diverses possibilités de supexposition des compétences favorisent en général l'extension des interventions de l'Etat. Celui-ci dispose, d'une part, des compétences résiduaires et, d'autre part, d'une maitrise complète de ses ressources financières. Sa prédominance se trouve d'autant mieux assise qu'il contrôle également de façon très large les ressources des Communautés et des Régions. Ces avantages s'avèrent particulièrement déterminants dans la mesure où l'argent, nerf de la guerre, est aussi très largement le nerf du pouvoir.

Le maintien d'une nette prédominance du pouvoir central dans le nouveau cadre constitutionnel belge entraîne des conséquences fondamentales. D'une part, il explique la persistance d'affrontements communautaires aigus au sein du gouvernement et du Parlement. D'autre part, il met davantage en évidence une carence essentielle de l'évolution de la Belgique vers un véritable système fédéral : l'absence d'une représentation équilibrée des composantes de l'Etat au sein du pouvoir fédéral. Dans le présent contexte, cet équilibrage pourrait garantir que le pouvoir central n'utilisera pas sa maftrise des relations internationales, sa suprématie fiscale ou sa tutelle financière au détriment éventuel d'une communauté.

Une orientation de ce type transparaît, par exemple; dans le principe de parité du Conseil des ministres imposé par l'article 86bis de la Constitution. Elle correspondait également, à une certaine époque, au souci des gouvernements d'obtenir une majorité dans les deux Communautés du pays, voire dans ses trois Régions. Cette aspiration s'est aussi traduite par la revendication d'un Sénat paritaire. Une solution alternative consisterait à étendre, dans la procédure législative, la technique des lois votées à la majorité de chaque groupe linguistique. L'exigence d'une majorité dans chaque groupe linguistique fournit aux deux communautés du pays une garantie sans doute meilleure que la création d'un Sénat paritaire. Il faut noter, en tout cas, qu'un Sénat paritaire ne présente guère d'intérêt s'il ne participe pas, d'une façon ou d'une autre, à la décision législative et, par voie de conséquence, à I'investiture des gouvernements. La parité au sein du Conseil des ministres constitue une garantie encore plus faible, indépendamment du problème posé par le Premier ministre. D'abord, elle ne tient pas compte des secrétaires d'Etat. Ensuite, un ministre peut cumuler un nombre de ministères plus ou moins importants. Enfin, les ministères n'ont pas tous le même poids. De tels mécanismes vont à l'encontre de la règle majoritaire. Mais là réside le fondement même du principe fédéral : la protection des minorités résulte du fait 
que l'action du pouvoir central requiert davantage qu'une simple majorité. Aux Etats-Unis, il arrive que la majorité de la Chambre s'oppose à la majorité du Sénat. En République fédérale d'Allemagne, le Bundesrat met parfois des obstacles a la politique soutenue par. le Bundestag. Il s'agit d'une conséquence inhérente au système fédéral. De façon paradoxale, l'octroi d'une telle garantie a souvent contribué à une centralisation progressive.

Malgré quelques éléments embryonnaires, la Belgique se situe encore fort loin de ce modele. Certains affirment que la règle de la majorité a déjà vécu en Belgique (30). La constatation ne parait pas fondée. La multiplication, au cours des dernières années, des votes opposant une communauté à l'autre au sein du Parlement tend à démontrer l'inverse (31). Le recours à la majorité qualifiée, ou plus exactement à l'exigence d'une approbation majoritaire dans les deux grandes communautés du pays, n'a pas été suffisamment développé.

L'élaboration d'une véritable structure fédérale se heurte toutefois à un obstacle de taille en Belgique. Vraisemblablement, cette structure ne comprendrait que deux composantes. Cela pose un problème dans la mesure où il n'existe pas de stade intermédiaire entre la règle de la majorité simple et la règle de la majorité dans toutes les composantes du' système. Une telle solution intermédieire requiert en effet un plus grand nombre de composantes, comme aux Etats-Unis, en République fédérale d'Allemagne ou en suisse. Cela explique pourquoi les systèmes fédéraux à deux composantes demeurent très peu fréquents. Les rares exemples connus, à savoir Chypre et le Liban, n'offrent d'ailleurs pas des perspectives excessivement engageantes.

Pour surmonter cet obstacle, on a parfois évoqué la perspective d'un fédéralisme provincial. Cette solution, toutefois, ne résiste guère à l'examen. Les clivages linguistiques sont tellement enracinés dans la réalité sociologique belge qu'il parait vain d'espérer les effacer en divisant les régions flamande et wallonne chacune en cing provinces.

Une représentation plus équilibrée des deux principales commu-

(30) Sur ce thème, volr L. Neels, "De verdelling van de natlonale en reglonale bevoegdheden", Res Publica, 1984, pp. 365-366.

(31) Dans la plupart des cas, ces votes ont porté sur les modifications envisagées aux lols llingulstiques, ou sur les procédures de conflits d'intérêt entre l'Etat, les Communautés ot les Réglons. 
nautés au sein du pouvoir central présente un intérêt d'autant plus grand qu'il existe, au sein des institutions nationales, une deuxième réforme de l'Etat. Les multiples imputations communautaires et régionales dans les budgets nationaux de la recherche, des communications ou des travaux publics, comme la dérive progressive des politiques "nationales" de l'enseignement le prouvent. L'instauration d'une politique différenciée et de conseils ministériels régionaux dans la gestion des secteurs "nationaux" le confirme (32).

Plus généralement, les dernières années ont montré combien la gestion de tous les départements nationaux avait des incidences communautaires. L'établissement des cadres linguistiques dans les administrations, la représentation belge dans les négociations avec la Commission européenne, menée conjointement par huit ministres lors des restructurations dans la sidérurgie, l'application plus ou moins rigoureuse des lois fiscales dans le nord et le sud du pays, les commandes publiques d'équipements militaires ou de systèmes de télécommunications reviennent régulièrement au premier plan de l'actualité. Plus fondamentalement, si les communautés ou les régions disposent d'appareils de production différents, la définition de la politique économique nationale présente une importance essentielle. Franc fort et franc faible, politique d'exportation et déficit budgétaire n'ont pas des retombées identiques partout.

(32) Sur ces problèmes, volr F. Dehousse, "Les confllts budgétalres ...", op. cit., pp. 48-68. 


\section{Conclusions}

La réforme de l'Etat belge : beaucoup de bruit pour rien? A cette question, il convient d'apporter une réponse particulièrement nuancée. Du point de vue conceptuel, la réforme de l'Etat présente indéniablement plusieurs caractéristiques novatrices : l'équipollence des normes, l'absence de tutelle nationale sur les Communautés et les Régions, l'exclusivité des compétences de ces dernières, la création de nouvelles assemblées parlementaires et de plusieurs exécutifs autonomes vis-à-vis du gouvernement national. Du point de vue pratique, la mutation se révèle cependant beaucoup moins fondamentale.

Cette contradiction reflète les contradictions de la société belge. Quoiqu'il ait été reconnu dès 1970 que "l'Etat unitaire est dépassé par les faits", il n'existe toujours pas d'accord sur une structure alternative. D'où les incohérences, multiples d'une réforme où les matières ont été divisées afin de donner satisfaction à tout le monde et où, en quelque sorte, la centralisation sort par. la porte pour rentrer par la fenêtre. La réforme de l'Etat belge instaure un système sui generis : du point de vue formel, elle revêt des apparences fédérales mais, au fond des choses, il s'agit d'une décentralisation encadrée par plusieurs formes indirectes de tutelle.

Ces incohérences ne doivent pas être attribuées, comme on le fait parfois, à une impréparation de la réforme de l'Etat : comparativement, la Constitution de 1831, plus fondamentale encore, a été adoptée beaucoup plus vite. Elles résultent du fait que les compromis politiques dont la réforme de l'Etat émane n'ont pas été réalisés dans la clarté. Ceci explique la multiplication des règles de compétence. Cette multiplication a permis d'affirmer à la fois une chose et son contraire, d'attribuer une apparence de souveraineté aux Communautés et aux Régions tout en préservant certains mécanismes de tutelle indirecte. Il arrive que l'inflation résulte du souci de satisfaire toutes les revendications économiques; ici, l'inflation des normes provient du souci de satisfaire toutes les revendications politiques. 
Aux fondements du fédéralisme se trouve l'idée d'un pacte (foedus) et d'une souveraineté partagée entre plusieurs niveaux de pouvoir. Ce partage de souveraineté peut s'effectuer de nombreuses manières; néanmoins, certains principes demeurent constants. Les différents niveaux de pouvoir détiennent chacun certaines compétences et certaines ressources. Ils coopèrent entre eux, mais conservent toujours une autonomie réelle. Leurs conflits sont arbitrés par un organe à la désignation duquel ils participent. Ils interviennent également dans la révision des textes constitutionnels définissant la structure et le fonctionnement du système.

La réforme de l'Etat en Belgique présente plusieurs carences par rapport à ce modèle. L'Etat intervient malgré tout-quelque peu dans la désignation des exécutifs communautaires et régionaux. Les Communautés et les Régions ne disposent pas des compétences ni des moyens requis pour garantir leur autonomie réelle vis-à-vis de l'Etat. Elles n'interviennent mullement dans la désignation de l'organe chargé d'arbitrer les conflits. Elles ne bénéficient pas d'une représentation équilibrée au sein de l'Etat.

L'absence de consensus sur la nouvelle structure de l'Etat a des conséquences graves. Elle encourage les interprétations divergentes et les conflits de compétence. Elle stimule les tendances centrifuges, les représentants flamands. espérant ainsi se soustraire à certains mécanismes de transfert défavorables et les représentants francophones échapper au poids de plus en plus pesant de la majorité. Mais, avant tout, l'absence de consensus paralyse l'administration quotidienne du pays. Le recours permanent à la "globalisation" des actes réglementaires, des nominations et des commandes publiques complique la gestion gouvernementale et réduit son efficacité. Si la politique trouve son compte dans des solutions de dernière minute fondées sur le pourrissement et l'amalgame des. problèmes les plus divers, on ne peut en dire autant de l'Etat, des entreprises et des particuliers.

Si la réforme de l'Etat se poursuit en fonction de cette orientation sui generis actuelle, les blocages politiques actuels subsisteront, selon toute vraissemblance. La concentration des responsabilités au sein du gouvernement et la perte d'autonomie individuelle des ministres s'accentueront. Plus fondamentalement, si on persiste a transférer aux Communautés et aux Régions les matières dont la gestion entraîne des blocages au sein de l'Etat sans donner une solution structurelle à ces blocages, l'Etat se réduira bientôt à la gestion des dettes du passé. A moins d'envisager sa liquidation, il importe de dégager des règles de fonctionnement viables et 
acceptables par les deux principales communautés au niveau du pouvoir central.

Si, par contre, on souhaite instaurer une structure fédérale complète en Belgique, la représentation plus équilibrée des grandes communautés du pays au sein des institutions nationales constitue une nécessité. Là où ce principe a été instauré, au gouvernement ou à la Cour d'arbitrage, il a favorisé l'émergence de compromis. Son extension devrait être envisagée dans l'enceinte du Parlement. Le principe devrait également être appliqué davantage au sein du Conseil d'Etat ou de la Cour des comptes. L'expérience montre en effet qu'une scission interne trop poussée de ces institutions peut aboutir à des dérapages d'interprétation menaçants pour l'unité du système juridique belge.

Plusieurs autres réformes devraient en outre être envisagées. Les compétences et les ressources des Communautés et des Régions devraient être accrues, afin qu'elles deviennent davantage qu'un simple accessoire des politiques nationales. Les compétences devraient être rationalisées en regroupant certaines matières découpées à l'excès (l'audiovisuel et la publicité commerciale à la radio et à la télévision, par exemple, les programmes et les diplômes dans l'enseignement, l'initiative industrielle publique et les aides d'expansion dans la politique industrielle, etc.). Les ressources des Communautés et des Régions devraient être définies dans une loi à majorité spéciale. Le statut de la Cour d'arbitrage devrait également être fixé dans une loi à majorité spéciale; la désignation de ses membres devrait revenir aux deux groupes linguistiques du Sénat plutôt qu'au Sénat dans son ensemble. Enfin, l'autonomie des différents niveaux de pouvoir devrait être consacrée en supprimant la possibilité des doubles mandats électifs et en séparant les élections nationales des élections concernant les institutions communautaires et régionales.

Malgré ses nombreux défauts actuels, l'utilité de la réforme de l'Etat ne doit pas etre sous-estimée. Les affrontements constants dans les institutions nationales démontrent la nécessité d'une laxge dévolution de compétences et de moyens aux Régions et aux Communautés. Les litiges communautaires compliquent la gestion des dossiers mais, en outre, ils obscurcissent leurs implications essentielles. Le côté passionnel des débats, une tendance naturelle à rejeter les responsabilités sur autrui occultent les enjeux réels.

Par ailleurs, malgré sa fragilité, la portée de la réforme de l'Etat ne doit pas être sous-estimée. La possibilité d'une 
réduction substantielle, voire d'une suppression des moyens financiers des Communautés et des Régions existe en théorie. Toutefois, peut-on réellement envisager une pareille hypothèse? Aux Etats-Unis, des observateurs de la Constitution ont remarqué avec justesse que le Congrès pouvait réduire les pouvoirs du Président à leur plus simple expression rien qu'en refusant les crédits requis par la Maison Blanche. Après deux cents ans, cette possibilité n'a pas encore reçu la moindre concrétisation.

De même, on imagine mal la suppression complète, par la voie financière, des institutions communautaires et régionales. Malgré leurs ambiguités, ces institutions correspondent à une évolution des mentalités dans les différentes parties de la Belgique. Tant que ces mentalités ne changeront pas, les Communautés et les Régions subsisteront, selon toute vraisemblance, en dépit de la précarité de leur statut juridique. Sur ce terrain également, la comparaison avec les Etats-Unis se révèle éclairante. Si le fédéralisme américain demeure étonnamment vivace après tant d'altérations successives, c'est avant tout parce qu'il repose sur une réalité sociologique profonde. A cet égard, le juriste doit bien reconnaître que la sociologie fournit peut-être de meilleures garanties que le droit. 